\title{
На пути к протологике ${ }^{1}$
}

\author{
A. C. КАРПЕНКО
}

\begin{abstract}
This paper is devoted to the problem of the foundations of logic. The path of development of logic towards generalization and abstraction is traced. The central notions here are minimal logic, basic logic, abstract logic, universal logic, protologic.

Ключевые слова: минимальная логика, базисная логика, абстрактная логика, универсальная логика, протологика
\end{abstract}

\section{1 Введение}

Ровно через сто лет после выхода в 1879 г. в свет знаменитой работы Г. Фреге «Bergiffsschrift» [12], в которой вводятся предикаты, отрицание, условная связь (the conditional) и кванторы как основа логики, а также введена идея формальной системы, в которой демонстрации должны осуществляться посредством явно сформулированных синтаксических правил, после ста лет триумфального развития логики как самостоятельной науки вдруг появляется статья Я. Хэкинга под названием «Что есть логика?» [35]. В ней высоко оценивается введение Г. Генценом структурных правил, работа с которыми позволяет выражать те аспекты логических систем, которые не имеют непосредственного отношения к логическим константам, ставится вопрос о логических константах и четко обозначается смена парадигмы от изучения истин к теоретико-доказательным методам, которые, в свою очередь, и являются основанием для введения логических операций. Основная идея Хэкинга заключается в том, что логические системы можно представлять поразному.

Статья Хэкинга переиздается и открывает собой большой сборник работ под названием «Что есть логическая система?» [32], который издается в Англии и Америке. Заметим, что

\footnotetext{
${ }^{1}$ Работа выполнена при поддержке РГНФ, грант № 11-03-00761a.
} 
в этой книге также опубликована статья Дж. Ламбека под названием «Что есть дедуктивная система?» [40], в которой выделяется пять стилей дедуктивных систем. В этом же году и с тем же названием, что и статья Хэкинга, публикуется философская работа Хао Вана [49], которая открывается определениями логики, начиная от Канта и вплоть до Гёделя и заканчивается характеризацией логики, данной Л. Витгенштейном в 1921 г. в его "Трактате. ..": «Логика трактует каждую возможность, и все возможности суть ее факты (2.0121)» [1]. Интересно, что в этом же 1994 году под названием «Что есть истинная элементарная логика?» появляется статья выдающегося логика и философа Яакко Хинтикки [37], в которой развивается новая концепция первопорядковой логики (см. также его совместную статью с Г. Санду «Революция в логике?» [38]). К работам Я. Хинтикки мы еще вернемся.

Ввиду сказанного нас интересует следующая проблема: почему после столь бурного и триумфального развития современной логики, начиная с работ Дж. Буля (середина XIX в.), вдруг в научной литературе в конце ХХ в. стал активно обсуждаться вопрос: «Что есть логика»? (см. также [5] и [39]). Сам этот вопрос говорит о кризисе в основаниях логики, приведшем к полной переоценке самого статуса логики и её назначения. Интересен анализ причин произошедшего.

\section{2 Кризис в основаниях логики}

Стоит заметить, что традиционный подход к пониманию логики весьма подкупает тем, что логику в нем можно попытаться определить посредством совокупности логических законов, ее задающих. Тогда одной из причин кризиса оснований логики является критика основных законов логики ${ }^{2}$, предпринятая еще в начале XX в. Л. Брауэром (закон исключенного третьего), Н.А. Васильевым и Я. Лукасевичем (закон непротиворечия). Результатом стало появление различных систем интуиционистских и паранепротиворечвых логик. Затем подверглись критике основные свойства материальной (классической) импликации К. Льюисом (1912) и свойства строгой импликации В. Аккерманном (1956).

\footnotetext{
${ }^{2}$ См. в [6] раздел «Содержательная критика основных законов и принципов классической логики высказываний».
} 
В результате появились многочисленные системы модальных и релевантных логик.

Подчеркнем, что критика основных законов классической логики продолжалась целое столетие и приняла тотальный характер, когда, казалось, совсем незыблемые законы были отвергнуты. Так, был отвергнут импликативный закон идентичности $A \supset A$, поскольку, согласно Э. Шрёдингеру, этот закон в общем случае не имеет места для микрообъектов. Такие логики получили название «логики Шрёдингера» [30]. Также не выдержал испытания и импликативный закон сильной транзитивности $(A \rightarrow B) \rightarrow((B \rightarrow C) \rightarrow(A \rightarrow C))$ [43]. Из всего этого следует, что если сделать теоретико-множественное пересечение логических систем относительно верификации законов классической логики, то в результате получим пустое множество. Отсюда можно сделать один очень важный вывод: не существует какой-либо выделенной системы логики. Хотя время от времени появлялись и появляются все новые логики, каждая из которых представляет особый интерес в силу своих приложений или металогических свойств ${ }^{3}$.

Главным следствием всего этого процесса стало появление огромного класса новых логических систем. Было установлено, что существуют бесконечные классы логик, а в большинстве случаев эти классы континуальны. Первый результат в этой области был получен российским логиком В.А. Янковым [15], где было показано, что мощность класса суперинтуиционистских логик есть континуум. Более того, оказалось, что континуальность классов логик является не исключением, а нормой [3]. В связи с этим обратим внимание на главнейшую тенденцию развития современной логики, которая заключается в том, что

\footnotetext{
${ }^{3}$ Стоит заметить, что в последнее время такой логикой стала система, созданная Я. Хинтиккой и названная им IF-логикой (см. [37], а также [38]). IF-логика представляет собой расширение классической логики предикатов за счет введения ветвящихся кванторов. Следствием этого явилась ее семантическая неполнота. Таким бразом, IF-логика не является аксиоматизируемой. Но в то же время она удовлетворяет свойствам компактности и Лёвенгейма-Скулема, а также обладает интерполяционным свойством. Несмотря на это $\mathbf{I F}$-логика по своим выразительным возможностям очень богата и сравнима со второпорядковой логикой. В силу таких ее возможностей Хинтикка считает эту логику наиболее пригодной для решения проблем оснований математики.
} 
изучается не какая-то специфическая логическая система, какой бы она ни была интересной, а подвергается исследованию некоторый класс логик в виде определенной структуры ${ }^{4}$.

В итоге главная проблема, порожденная кризисом в основаниях логики, может быть сформулирована следующим образом: Имеется ли все-таки одна «истинная» логика и если нет, то как мы можем ограничить наше понимание логики или, более конкретно, понимание логической системы. Обратим внимание, что статья А. Тсарпа так и называется: «Какая логика является правильной логикой?» [46]. Видимо, стоит согласиться с Дж. ван Бентемом и К. Дэтсом [48, р. 326], что «никакая специфическая теория не является священной в современной логике». Учитывая исключительное развитие неклассических логик, стоит констатировать, что подобный ответ является вызовом для всех логиков-исследователей. Тогда можно поставить вопрос следующим образом: «Как логика в лице своих известных представителей отвечает на этот вызов?»

\section{3 Минимальная логика}

Понятие минимальной логики имеет смысл в некотором хорошо определенном классе неклассических логик. Например, в классе модальных льюисовских систем таковой является модальная логика $\mathbf{K}$, которая получается посредством добавления к классической логике лишь аксиомы

$$
\square(A \rightarrow B) \rightarrow(\square A \rightarrow \square B) .
$$

Иногда логика $\mathbf{K}$ называется базисной в силу того, что в семантике Крипке для модальных логик на отношение достижимости не накладывается никаких ограничений. Другими словами, множество формул логики $\mathbf{K}$ общезначимо во всех шкалах Крипке (см. [24]). Другим примером является минимальная временная логика $\mathbf{K}_{\mathbf{t}}$, которая получается за счет добавления к классической логике минимального числа аксиом с временными операторами (см. [4]).

Но нас больше интересует тенденция в сторону ограничения логических законов до их некоторого минимума. Уже на самом

\footnotetext{
${ }^{4}$ Например, в [26] глава 4 называется «От логик к классам логик».
} 
раннем этапе четко обозначилась тенденция попытаться определить систему «минимальной логики». Уже в 1936 г. И. Йохансон определила «минимальное исчисление», которым оказалось ослабление интуиционистской логики $\mathbf{H}$ за счет отбрасывания закона Дунса Скота $A \rightarrow(\neg A \rightarrow B)$ : «из противоречия следует все что угодно». Эта система обозначается посредством $\mathbf{J}$ и может быть аксиоматизирована следующим образом: к позитивной логике Гильберта ${ }^{5}$ добавляется аксиома

$$
((A \rightarrow B) \&(A \rightarrow \neg B)) \rightarrow \neg A .
$$

Стоит отметить, что А.Н. Колмогоров в 1925 г. в знаменитой статье «О принципе tertium non datur» [7]), разделяя критику закона исключенного третьего Л. Брауэром, подвергает также критике закон Дунса Скота. В результате строится первое в мире предикатное паранепротиворечивое исчисление (импликативно-негативное $)^{6}$. Тогда пропозициональную минимальную логику Колмогорова можно аксиоматизировать следующим образом. $\mathrm{K}$ импликативному фрагменту позитивной логики Гильберта, который обозначим посредством $\mathbf{H}_{\rightarrow}$ :

$$
\begin{aligned}
& \text { K. } A \rightarrow(B \rightarrow A) \\
& \text { S. }(A \rightarrow(B \rightarrow C)) \rightarrow((A \rightarrow B) \rightarrow(A \rightarrow C))^{7},
\end{aligned}
$$

добавляется закон приведения к абсурду

$$
((A \rightarrow B) \rightarrow((A \rightarrow \neg B) \rightarrow \neg A) .
$$

Заметим, что для логик, содержащих в качестве фрагмента

\footnotetext{
${ }^{5}$ Позитивная логика введена Гильбертом в 1934 г. (см. [2, с. 99]) и представляет собой такую систему логики, что добавление к ней закона Пирса $((A \rightarrow B) \rightarrow A) \rightarrow A$ дает позитивный фрагмент пропозициональной классической логики.

${ }^{6}$ См. также [9].

${ }^{7}$ Логика $\mathbf{H}_{\rightarrow}$ под названием «позитивная импликативная логика» была введена Гильбертом в 1922 г. и представляет собой следующую систему аксиом, эквивалентную приведенной:
B. $(B \rightarrow C) \rightarrow((A \rightarrow B) \rightarrow(A \rightarrow C))$
C. $(A \rightarrow(B \rightarrow C)) \rightarrow(B \rightarrow(A \rightarrow C))$
W. $(A \rightarrow(A \rightarrow B) \rightarrow(A \rightarrow B)$
K. $A \rightarrow(B \rightarrow A)$ 
$\mathbf{H}_{\supset}$, имеет место стандартная теорема дедукции. Отметим также, что удивительным образом простейшие типы для базовых комбинаторов соответствуют аксиомам логики $\mathbf{H}_{\rightarrow}[28]$. Это так называемый изоморфизм Карри-Ховарда, который устанавливает, что полное множество исходных комбинаторов (например, $\mathbf{B}$, $\mathbf{C}, \mathbf{W}, \mathbf{K}$ ) определяет собой импликативный фрагмент интуиционистской пропозициональной логики, т.е. $\mathbf{H}_{\rightarrow}$.

Известно, что комбинаторная логика тесно связана с $\lambda$-исчислением, построенным А. Чёрчем в начале 1930-х годов. Таким образом, имеется изоморфизм между $\mathbf{H}_{\rightarrow}$ и типизированными замкнутыми $\lambda$-термами. В итоге можно было бы считать, что логика $\mathbf{H}_{\rightarrow}$ является прекрасным кандидатом на роль минимальной логики.

Однако в 1951 г. появляется статья А. Чёрча «Минимальная логика» [25], где строится «слабое позитивное импликативное исчисление». Эта логика получается из $\mathbf{H}_{\rightarrow}$ посредством замены формулы $A \supset(B \supset A)$ на формулу $A \supset A$, которой соответствует комбинатор I. Впоследствии оказалось, что полученная логика является импликативным фрагментом релевантной логики $\mathbf{R}$ (эта логика стала обозначаться посредством $\mathbf{R}_{\rightarrow}$, т.е. $\mathbf{R}_{\rightarrow}$ есть $\left.\mathbf{B}, \mathbf{C}, \mathbf{W}, \mathbf{I},\right)$. Для этой логики Чёрчем была доказана ослабленная теорема дедукции и было установлено соответствие между $\mathbf{R}_{\rightarrow}$ и исчислением $\lambda \mathrm{I}$.

В 1956 г. К.А. Мередит вводит систему B, C, I, при этом также обращая внимание на соответствие между импликативными формулами и комбинаторами. Обратим внимание на то, что в последнее время получила развитие так называемая линейная логика, введенная Дж. Жираром [33] и нашедшая широкое применение в компьютерных науках. Ее взаимоотношение с другими логиками, и в особенности с релевантными, исследованы А. Авроном [18]. В этой работе $\mathbf{H L} \rightarrow$ обозначает импликативный фрагмент данной линейной логики, который в точности совпадает с аксиомами $\mathbf{B}, \mathbf{C}, \mathbf{I}$. Аврон следующим образом определяет $\mathbf{H L}_{\rightarrow}$ : Это «минимальная система, соответствующая линейному доказательству», которое, в свою очередь, определяется так: «Линейное доказательство есть классическое доказательство, в котором каждое вхождение формулы, отличной от последней, используется в точности один раз в качестве посылок МР». 
Исходя из идеи Чёрча о минимальной логике, Х. Карри [27] обобщает теорему дедукции для логики IB. Таким образом, импликативную логику IB можно было бы считать минимальной логикой. Идея о минимальной логике оказалась очень близкой специалистам в области релевантной логики. А. Уркварт [47, p. 168] формулирует «минимальную импликативную логику в смысле Чёрча», которая отличается от IB тем, что кроме правила МР имеется также правило: из $A$ следует $(A \rightarrow B) \rightarrow B$. Характерной чертой этой логики, замечает Уркварт, является отсутствие структурных правил при ее секвенциальной формулировке. Интересно, что в этом же году импликативная логика с аксиомами I и В была рассмотрена также В.А. Смирновым [11, c. 72-74], при этом доказана теорема дедукции, которая имеет место при наличии следующей теоремы: «если существует доказательство формулы $D$, то существует доказательство формулы $(A \rightarrow(D \rightarrow B)) \rightarrow(A \rightarrow B) »$. А. Андерсон и Н. Белнап в $[16$, $\S 8.11]$ в качестве минимальной логики предложили считать систему IBB' (с правилом МР), где аксиома $\mathbf{B}^{\prime}$ есть закон сильной транзитивности (см. выше). Тема получила дальнейшее развитие в [17, p. 149], где таковой объявляется логика, предложенная А. Урквартом. Эту логику Андерсон и Белнап обозначали посредством $\mathbf{M}_{\rightarrow}$. Для нее строится модель $<S, v>$ такая, что $S$ есть ассоциативный моноид, и множество всех формул, истинных во всех $<S, v>$-моделях, аксиоматизируется посредством логики $\mathbf{M}_{\rightarrow}$.

Поскольку понятие моноида удовлетворяет условиям быть категорией, то данное понятие минимальной логики также обладает данными свойствами. Другими словами, дедуктивная система, отношение следования (дедуцируемости) которой рефлексивно и транзитивно, является категорией. Поэтому с полным основанием логику $\mathbf{M}_{\rightarrow}$ можно было бы считать минимальной логической системой. Однако не очень понятно, почему в основе логических рассуждений должна лежать структура ассоциативного моноида? Более того, К. Дошен в качестве минимальной логики предлагает считать неассочиативное исчисление Ламбека с единственной бинарной связкой «мультипликации» [31, p. 20]. 


\section{4 Базисная логика}

Другая тенденция заключается в выборе некоторой базисной логики. Однако сразу стоит сказать, что такой выбор является весьма произвольным и имеет смысл только для определенного класса логик. Например, в классе всех нормальных модальных логик таковой является логика $\mathbf{K}$. В качестве базисной логики предлагается также полное исчисление Ламбека FL. Грубо говоря, это интуиционистская логика без структурных правил (см. [42]). Кроме комбинирования FL со структурными правилами, соответствующими формулам $\mathbf{C}, \mathbf{W}$ и $\mathbf{K}$, к некоторым логикам добавляется еще закон снятия двойного отрицания $\neg \neg A \rightarrow A$. Интересной работой является [20], где вводится базисная логика $\mathbf{B}$ и строится ее секвенциальное исчисление ${ }^{8}$. Эта логика вообще не имеет структурных правил и может рассматриваться как «логика свлзок». Комбинирование последней осуществляется с тремя независимыми свойствами $\mathbf{C}, \mathbf{D}$ и $\mathbf{S}$, где $\mathbf{C}$ есть правила для снятия двойного отрицания, $\mathbf{D}$ состоит из обычных свойств импликации, удовлетворяющих теореме дедукции, и $\mathbf{S}$ есть структурные правила, соответствующие формулам $\mathbf{W}$ и $\mathbf{K}$, плюс идентификация двух констант, выражающих ложь. Восемь возможных комбинаций свойств $\mathbf{C}, \mathbf{D}$ и $\mathbf{S}$ продуцируют куб логик. В частности, добавление $\mathbf{C}$ и $\mathbf{D}$ к $\mathbf{B}$ дает линейную логику Жирара, добавление $\mathbf{D}$ и $\mathbf{S}$ дает интуиционистскую логику, добавление $\mathbf{C}$ и $\mathbf{S}$ дает ортологику и, наконец, добавление $\mathbf{C}, \mathbf{D}$ и $\mathbf{S}$ дает классическую логику.

Последнее время распространение получила базисная нечеткая логика BL [36], которая получается за счет ослабления бесконечнозначной логики Лукасевича ${ }^{9}$.

\footnotetext{
${ }^{8}$ Эта логика введена в 1995 г.

${ }^{9}$ Ради полноты изложения приведем формулировку базисной логики, введенной А. Авроном в [19]. Это позитивный фрагмент классической пропозициональной логики $\mathbf{C}_{2}$, плюс законы де Моргана и аксиомы $\sim A \approx A$, $\sin (A \Rightarrow B) \approx A \wedge \sim B$. Впервые аксиоматизация этой логики появилась в [10], где она обозначена посредством Par. Если теперь добавить $A \vee \sim A$, то получим в точности аксиоматизацию трезначной паранепротиворечивой логики Pcont. Если же добавить $A \rightarrow(\sim A \rightarrow B)$, то получим аксиоматизацию трехзначной логики, которая функционально эквивалентна трехзначной логике Лукасевича $\mathbf{Ł}_{3}$. Если же добавим одновременно обе эти формулы, продолжает А. Аврон, то получим аксиоматизацию классической логики $\mathbf{C}_{2}$.
} 
Таким образом, смысл обоих указанных подходов, а именно выбор минимальной или базисной логики, заключается в том, что предлагается какой-то минимум логических средств и затем посредством различных расширений получаются известные логические исчисления вплоть до самой классической логики. На самом деле вся исходная парадигма неверна: в основу берется классическое пропозициональное исчисление, и затем добавляются или, как мы видели, отбрасываются те или иные законы этой логики. Но остается открытым вопрос об обосновании полученного минимума или базиса логических средств, не говоря уже об обоснованности самого корпуса классической логики. Если делаются какие-то предпосылки, то на чем они основаны? В идеале хотелось бы вычленить какое-то логическое «ядро», не основываясь ни на каких жестких предпосылках.

\section{5 Абстрагирование и обобщение}

Пока мы рассмотрели философскую сторону дела. Однако параллельно с указанными тенденциями в недрах математической логики развивалась тенденция в сторону максимального обобщения или абстрагирования понятия логики.

В польской школе логиков получило распространение определение пропозициональной логики, введенное А. Тарским в 1930 г. (см. [44], [45]). Пусть $\mathcal{P}(A)$ есть множество всех подмножеств множества $A$. Оператором замыкания на множестве $A$ называется отображение $C: \mathcal{P}(A) \rightarrow \mathcal{P}(A)$, которое удовлетворяет следующим условиям для каждого $X, Y \subseteq A$ :
(C1) $\quad X \subseteq C(X)$
(рефлексивность),
(C2) $C C(X)=C(X)$
(идемпотентность),
(С3) Если $X \subseteq Y$, то $C(X) \subseteq C(Y)$ (монотонность).

Подмножество $X$ из $A$ называется замкнутым подмножеством, если $C(X)=X$.

А. Тарский [44] находит удивительное применение оператору замыкания для изучения абстрактного отношения следования. Пусть $A$ есть множество всех формул $F m$ в пропозициональном языке $\mathcal{L}$. Тогда оператор замыкания $C$ называется операцией присоединения следствий (consequence operation) и обозначается посредством $C n$, т.е. $C n$ есть операция, которая, примененная 
к множествам формул, позволяет получать новые множества формул. Пусть $X$ есть множество предложений, тогда $C n(X)$ обозначает множество всех предложений, которое может быть дедуцировано их $X$. Операция присоединения следствий $C n$ называется структурной (или инвариантной относительно подстановки), если для всех подстановок $e$ (эндоморфизмов) пропозиционального языка $\mathcal{L}$ выполняется условие

$$
\text { (C4) } e(C n(X)) \subseteq C n(e(X)) \text {. }
$$

Под логикой (пропозициональной) понимается пара $<F m$, $C n>$, где абстрактная операция присоединения следствий $C n$ является структурной. Изучению основных свойств операции присоединения следствий посвящена фундаментальная монография Р. Вуйцицкого [51]. Связь между операцией $C n$ и обычным отношением выводимости $\vdash$ очевидна:

$A_{1}, A_{2}, \ldots, A_{n} \vdash B$ тогда и только тогда, когда $\mathrm{B} \in C n\left(\left\{A_{1}, A_{2}\right.\right.$, $\left.\left.\ldots, A_{n}\right\}\right)$.

В 1969 г. Р. Вуйцицкий вводит понятие «обобщенной логической матрицы» [50]. Обобщенная матрица есть пара $\mathcal{A}=$ $<\mathbf{A}, C>$, где $\mathbf{A}$ есть алгебра соответствующего типа и $C \subseteq$ $\mathcal{P}(\mathbf{A})$ есть произвольное семейство подмножеств А. Обобщенные матрицы имеют хорошо известное дуальное представление как пара $\langle\mathbf{A}, C\rangle$, где $C$ есть оператор замыкания (см. выше). Специально обобщенным матрицам, их применению и развитию посвящена статья [34]. Наконец что в известной статье Д. Брауна и Р. Сушко [23] вводится термин «абстрактная логика» $\mathbf{L}=<\mathbf{A}, C>$, где $\mathbf{A}$ есть абстрактная алгебра, а $C$ есть абстрактная операция присоединения следствий (без структурности).

Под влиянием этих работ в 1994 г. ЖК.-И. Безьё вводит понятие «универсальной логики» [21] (см. также его же исторический экскурс [22]). По замыслу Безьё универсальная логика имеет такое же отношение ко всем конкретным логикам, как универсальная алгебра к конкретным алгебрам. Универсальная логика подобно универсальной алгебре есть часть общей теории структур и поэтому центральный вопрос заключается в следующем: какого вида структуры являются логическими структурами? В качестве ответа Безьё идет по пути дальнейшего обобщения тео- 
рии Тарского об операторе присоединения следствий. В результате под логической структурой понимается пара $<\mathbb{S}, \vdash>$, где первым членом является некоторая структура без всякой спецификаци, т.е. $\mathbb{S}$ не обязательно является алгебраической структурой, а вторым членом является отношение следования $\vdash$, где $\vdash$ есть подмножество $\mathcal{P}(\mathbb{S}) \times \mathbb{S}$. Заметим, что в отличие от оператора $C n$ на $\vdash$ не накладывается никаких ограничений.

Обратим внимание, что параллельно с этим в среде прикладной логики все большее распространение стало получать представление логики в виде функииональной системы ${ }^{10}$, т.е. логика есть пара $\left\langle P_{n}, C>\right.$, где $P_{n}$ есть множество $n$-значных функций, а $C$ есть операция суперпозиции, определенная на этом множестве. Если вместо $P_{n}$ берем множество булевых функций, то получаем модель для классической пропозициональной логики. Заметим, что множество всех функций, которые могут быть получены из функций системы $\mathfrak{R}$ с помощью операции суперпозиции, называется замыканием $\mathfrak{R}$ и обозначается посредством [R] . Очевидно, что операция [] обладает указанными выше свойствами $(\mathrm{C} 1)-(\mathrm{C} 3)$. Таким образом, здесь мы имеем еще один пример конкретизации оператора замыкания $C$.

Однако подчеркнем, что все эти подходы, развиваемые в русле абстрагирования, обобщения и универсализации понятия логики, обладают одним и тем же серьезным недостатком: в основе лежат очень сильные допущения. Так, в основе абстрактной логики и логики как функциональной системы лежит различная конкретизация топологического оператора замыкания, а в основе универсальной логики - обобщение этого оператора. В начале XX в. идеи топологии были очень популярны в среде польских математиков и логиков, но это не значит, что свойства данного оператора замыкания должны детерминировать некоторое «ядро» человеческих рассуждений.

\section{6 Протологика}

Итак, возникает естественный вопрос, а можно ли выделить некоторое протологическое ядро, которое априорно лежит в ос-

\footnotetext{
${ }^{10}$ Начало положено работами российских математиков А.В. Кузнецова и С.В. Яблонского и далее развито В.Б. Кудрявцевым, С.С. Марченковым и др. См. [8] и [41].
} 
нове человеческих рассуждений и не основывается ни на каких труднообъяснимых допущениях. Для этого необходимо вначале выделить ту естественную среду, которая априорно детерминирует некоторые исходные (прото)схемы рассуждений. Такой средой является человеческий язык, в котором оформляются все добытые знания. Но при этом «мы должны отвлечься от всякой специфики конкретных языков и взглянуть на них лишь как на абстрактные знаковые системы» [14]. Только при таком подходе мы можем подойти к построению логики, освобожденной от всяческих онтологических и эпистемологических допущений.

Прорыв в этой области совершен работами В.И. Шалака (см., в особенности, [13]), где вводится понятие протологического следования: Из посылок $\Sigma=\left\{B_{1}, \ldots, B_{n}\right\}$ протологически следует выражсение $A$, если и только если существует правило $R$, позволяюшее на основании значений посылок $\Sigma$ определить значение выражсения $A$.

Дале строится протологика - система правил знаковых преобразований - аналогов логических умозаключений. Протологику можно представить как пару, где имеется множество абстрактных знаков, структурированное группоидной операцией.

Дедукция осуществляется с помощью следующих трех правил:

1. Правила введения констант.

2. Правила построения термов.

3. Правила замены на основе ранее принятых определений.

Оказалось, что протологика в строго определенном смысле полна относительно комбинаторной логики и $\lambda$-исчисления Чёрча, а это означает ни много, ни мало, что в протологике выразимы все эффективно вычислимые функции. Многие математические объекты оказались имплицитно содержащимися в абстрактных языковых структурах. Нет ничего удивительного, что пространственно разделенные культуры приходили к одним и тем же вычислительным математическим структурам. Им для этого не требовалось никакой развитой логики, а требовалось всего лишь владение собственным языком.

Вычислимость - главное свойство протологики. В итоге получаем реализацию программы Лейбница: рассуждения заменяются вычислениями. 
Для сравнения с различными «минимальными» и «базисными» логиками, рассмотренными выше, приведем натуральную формулировку протобулевой логики, т.е. протологики с истинностно-значными термами:

$$
\begin{array}{llll}
\text { N.1 } & \frac{\varnothing}{A \vee \neg A} & \text { N.3 } & \frac{A, B}{A \& B} \\
\text { N.2 } & \frac{A}{\neg A} & \text { N.4 } & \frac{\vdash A \equiv B, A}{B}
\end{array}
$$

Как известно, человеческие рассуждения зачастую являются противоречивыми и иррациональными. Приведенные выше четыре правила составляют рациональное ядро логики, априорно присущей человеческим существам, владеющим той или иной знаковой системой.

\section{Литература}

[1] Витгенштейн Л. Логико-философский трактат. М.: Иностранная Литература, 1958.

[2] Гилъберт Д., Бернайс П. Основания математики. Логические исчисления и формализация арифметики. М.: Наука, 1979.

[3] Горбунов И.А., Ръбаков М.Н. Континуальные семейства логик // Логические исследования. 2007. Вып. 14. С. 131-151.

[4] Иимуратов А.Т. Логические теории временных контекстов. Киев: Наукова Думка, 1981.

[5] Карпенко А.С. Логика на рубеже тысячелетий // Логические исследования. 2000. Вып. 7. С. 7-60.

[6] Карпенко А.С. Неклассические логики versus классической // Логикофилософские штудии. 2005. Вып. 3. С. 48-73.

[7] Колмогоров А.Н. О принципе tertium non datur // Математический сборник. 1925. Вып. 32. №4. С. 668-677. (Переиздано: А.Н. Колмогоров. Избранные труды. Математика и механика. М., 1985).

[8] Кудрявцев В.Б. О функциональных системах. М.: ВЦ АН СССР, 1981.

[9] Плиско В.Е. Исчисление Колмогорова как фрагмент минимального исчисления // Успехи математических наук. 1988. Вып. 43. №1. С. 79-91.

[10] Попов В.М. Секвенциальные формулировки паранепротиворечивых логических систем // Синтаксические и семантические исследования неэкстенсиональных логик. М.: Наука, 1989. С. 285-289.

[11] Смирнов В.А. Формальный вывод и логические исчисления. М.: Наука, 1972.

[12] Фреге Г. Исчисление понятий, язык формул чистого мышления, построенный по образцу арифметического // Фреге Г. Логика и логическая семантика. М.: Аспект Пресс, 2000. С. 65-142.

[13] Шалак В.И. Протологика: новый взгляд на природу логического // Диссертация на соискание ученой степени доктора философских наук. М.: ИФ РАН, 2010.

[14] Шалак В.И. Два подхода к построению логики // Логические исследования. 2011. Вып. 17. (См. данный сборник). 
[15] Янков B.A. Построение последовательности сильно независимых суперинтуиционистских пропозициональных исчислений // Доклады Академии Наук CCCP. 1968. Вып. 181. №1. C. 33-34.

[16] Anderson A.R. and Belnap N.D. Entailment: The Logic of Relevance and Necessity. Princeton Univer. Press, 1975.

[17] Anderson A. R., Belnap N. D. and Dunn J.M. Entailment: The logic of Relevance and Necessity. Vol. 2. Princeton Univ. Press, 1992.

[18] Avron A. The semantics and proof theory of linear logic // Theoretical Computer Science. 1988. Vol. 57. P. 161-184.

[19] Avron A. Natural 3-valued logics - characterization and proof theory // The Journal of Symbolic Logic. 1991. Vol. 56. №1. P. 276-294.

[20] Battilotti G. and Sambin G. Basic logic and the cube of its extensions // A. Cantini (eds.). Logic and Foundations of Mathematics. Dordrecht: Kluwer, 1999. P. 165-186.

[21] Béziau J.-Y. Universal logic // Childers T. and Majer O. (eds.) Logica' 94 Proceedings of the $8^{\text {th }}$ International Symposium. Prague, 1994. P. 73-93.

[22] Béziau J.-Y. From consequences operator to Universal logic: A Survey of general Abstract logic // J.-Y. Béziau (ed.). Logica Universalis. Birkhäuser Verlag, 2005. P. 3-17.

[23] Brown D.J. and Suszko R. Abstract logics // Dissertationes Mathematicae. 1973. Vol. 102. P. 7-41.

[24] Chagrov A. and Zakharyascnev M. Modal Logic. Oxford: Clarendon Press, 1997.

[25] Church A. The weak theory of implication // Kontrolliertes Denken, Untersuchungen zum Logikkalkul und der Logik der Einzelwissenschaften. Münich, 1951. P. 22-37. (Abstract: The weak positive implicational propositional calculus // The Journal of Symbolic Logic. 1951. Vol. 16. №3. P. 238.).

[26] Chagrov A. and Zakharyaschev M. Modal Logic. Oxford: Clarendon Press, 1997.

[27] Curry H.B. Generalization of the deduction theorem // Proceedings of International Congress of Mathematicians. 1954. Vol. 2. Amsterdam. P. 399-400.

[28] Curry H.B., Feys R. Combinatory Logic. Vol. I. Amsterdam: North-Holland Co., 1958.

[29] Curry H.B. Generalisation of the deduction theorem // Proceedings of the International Congress of Mathematicans. 1954. Vol. 2. Amsterdam. P. 399-400.

[30] Da Costa N.C.A. and Krause D. Schrödinger logics // Studia Logica. 1994. Vol. 53. P. 533-550.

[31] Dožen K. A historical introduction to substructural logics // K. Dožen and P. Schroeder-Heister (eds.) Substructural Logics. Oxford: Clarendon Press, 1993.

[32] Gabbay D. M. (ed.) What is a logical system? Oxford: Clarendon Press, 1994 (and New Ysork 1995).

[33] Girard J.Y. Linear logic // Theoretical Computer Science. 1987. Vol. 50. P. 1-102.

[34] Font J.M. Generalized matrices in abstract algebraic logic // V.F. Hendricks and J. Malinowski (eds.). Trends in Logic: 50 Years of Studia Logica. Dordrecht: Kluwer, 2003. P. 57-86.

[35] Hacking I. What is logic? // The Journal of Philosophy. 1979. Vol. 76. №6. (Reprinted in: [Gabbay (ed.), 1994]. P. 1-33).

[36] Hájek P. Metamathematics of Fuzzy Logic. Dordrecht: Kluwer, 1998.

[37] Hintikka J. What is true elementary logic? // K. Gavroglu, J. Stachel and M. Wartofsky (eds.). Physics, philosophy and the scientific community. Dordrecht: Kluwer, 1994. P. 301-326.

[38] Hintikka J. and Sandu G. A revolution in logic? // Nordic Journal of Philosophical Logic. 1996. Vol. 1. №2. P. 169-183.

[39] Karpenko A.S. Modern study in philosophical logic: Worldwide level and Russian science // Studies in Logic, Grammar and Rhetoric. 2008. Vol. 14. №27. P. 35-71. 
[40] Lambek J. What is a deductive system? // [Gabbay (ed.), 1994]. P. 141-159.

[41] Lau D. Function Algebras on Finite Sets: A Basic Course on Many-Valued Logic and Clone Theory. Berlin: Springer-Verlag, 2006.

[42] Ono H. Structural rules and a logical hierarchy // P.P. Petkov (ed.). Mathematical Logic. New York: Plenum Press, 1990. P. 95-104.

[43] Tennant $N$. The transmission of truth and the transitivity of deduction // D.M. Gabbay (ed.). What is a logical system? Oxford: Clarendon Press, 1994. P. 161-177.

[44] Tarski A. On some fundamental concepts of metamathematics // Tarski A. Logic, Semantics, Metamathematics. Papers from 1923 to 1938. Oxford: The Clarendon Press, 1956. P. 30-37 (2nd ed. Indianopolis, 1983).

[45] Tarski A. Fundamental concepts of the methodology of the deductive sciences // Tarski A. Logic, Semantics, Metamathematics. Papers from 1923 to 1938. Oxford: The Clarendon Press, 1956. P. 60-109.

[46] Tharp L. Which logic is the right logic? // Synthese. 1975. Vol. 31. P. 1-21.

[47] Urquhart A. Semantics for relevant logics // The Journal of Symbolic logic. 1972. Vol. 37. P. 159-169.

[48] Van Benthem J. and Doets K. Higher-order logic // Gabbay and Guenthner (eds.). Handbook of Philosophical Logic. Dordrecht: Reidel, 1983. Vol. I. P. 275-329.

[49] Wang Hao What is logic? // The Monist. 1994. Vol 77. №3. P. 261-277.

[50] Wójcicki R. Logical matrices strongly adequate for structural sentential calculi // Bulletin de l'Académie Polonaise des Sciencies. 1969. Classe 3. Vol. XVII. P. 333335.

[51] Wójcicki R. Theory of Logical Calculi: Basic Theory of Consequences Operations. Dordrecht: Reidel, 1988. 